\title{
TESTE DE INTELIGÊNCIA R1 - FORMA B E G36: EVIDÊNCIA DE VALIDADE DE CRITÉRIO
}

\author{
R1 - Forma B and G36 intelligence tests: \\ criterion validity evidences
}

\author{
Ana Paula Porto Noronha ${ }^{1}$ \\ Fermino Fernandes Sisto ${ }^{2}$ \\ Acácia A. Angeli dos Santos ${ }^{3}$
}

\section{Resumo}

Esta pesquisa estudou evidências de validade de critério, utilizando-se da comparação entre grupos contrastantes. Para tanto, participaram como sujeitos 78 alunos de Cursos de Educação de Jovens e Adultos, todos eles freqüentando o telecurso de segundo grau. Foram aplicados coletivamente os testes de inteligência R1 - Forma B e o G36. Os resultados indicaram que os grupos extremos formados pelo R1 - Forma B foram discriminados pelas pontuações do G-36, tanto no que diz respeito ao resultado geral, quanto em relação aos fatores 1 e 2 do R-1. Dessa forma, concluiu-se que os resultados encontrados alicerçam a evidência de validade de critério contrastante proposta.

Palavras-chave: Inteligência; Testes Psicológicos; Parâmetros Psicométricos.

\section{Abstract}

This research studied evidences of criterion-related validity, comparing two different groups. 78 students attending at the high school television courses to young and adults were studied. Two intelligence tests were applied collectively - the R1-Form B and the G-36. The results indicated that the two different groups formed by the R1-Form B were discriminated by the G-36 scores, as much concerning to the total scores, as much in relation to the factors 1 and 2 of the R-1. So, it was concluded that the found results support the proposed evidence of criterion-related validity by contrast.

Keywords: Intelligence; Psychological tests; Psychometric parameters.

1 Doutora em Psicologia: Ciência e Profissão pela PUC-Campinas; bolsista de produtividade do CNPq; docente da graduação e do Programa de Pós-graduação Stricto Sensu em Psicologia da Universidade São Francisco - Itatiba-SP.

2 Doutor em Pedagogía pela Universidad Complutense de Madrid; livre docente em Psicologia do Desenvolvimento pela UNICAMP; docente da graduação e do Programa de Pós-graduação Stricto Sensu em Psicologia da Universidade São Francisco Itatiba-SP.

3 Doutora em Psicologia Escolar e do Desenvolvimento Humano pela USP; docente da graduação e do Programa de Pósgraduação Stricto Sensu em Psicologia da Universidade São Francisco - Itatiba-SP. Endereço para contato: Rua Alexandre Rodrigues Barbosa, 45, Centro, Itatiba-SP, CEP 13.251-900.

E-mail: ana.noronha@saofrancisco.edu.br 


\section{Introdução}

A inteligência tem conquistado o interesse de pesquisadores desde o início da psicologia como ciência moderna. Especialmente no que se refere à avaliação da inteligência, seu início e desenvolvimento se deram ao mesmo tempo em que a Psicologia se estabelecia como área de conhecimento (Anastasi \& Urbina, 2000).

No que tange às concepções teóricas de inteligência, a perspectiva mais tradicional dentro da Psicologia foi proposta por Spearman em 1927. Este autor considerou que toda a atividade intelectual está expressa em um fator geral (g), comum a toda atividade mental e possuidor de uma índole fisiológica, um fator específico (s) associado a tarefas individualizadas, e fatores de grupo que englobariam aspectos da conduta humana que possuíssem comunalidade. Nesse sentido, a inteligência é concebida como entidade simples ou complexa de fatores, traços ou aptidões mentais, que compõem a mente humana (Almeida, 1988).

Thurstone (1938), entre outros, assumiu uma posição contrária à de Spearman e o primeiro autor, por meio de uma análise fatorial, propôs sete fatores, entendidos como aptidões mentais diferenciadas, a saber: compreensão verbal, fluência verbal, aptidão numérica, velocidade perceptiva, aptidão espacial, memória, raciocínio, dedução e indução. Por sua vez, Cattell (1971) assumiu a existência de dois fatores gerais, os quais denominou de Inteligência Fluida (Gf) e Inteligência Cristalizada (Gc). A fluida pode ser entendida como a capacidade para raciocinar em situações novas ou inesperadas, enquanto a cristalizada, como representante da profundidade e quantidade de conhecimentos e experiências adquiridos (Schelini, 2000). Mais tarde, Horn (1991) acrescentou à concepção Gf-Gc outras seis capacidades cognitivas, quais sejam, processamento visual, memória a curto prazo, armazenamento, recuperação a longo prazo e velocidade de processamento, rapidez para a decisão correta e processamento auditivo.

Por meio de vários estudos de meta-análise, Carroll (1993) diferenciou as capacidades em três diferentes camadas ou estratos, a saber, camadas específicas, camadas amplas ou geral e relativa a uma única camada geral. Estudos mais recentes (Flanagan, McGrew \& Ortiz, 2000) propõem a integração entre os modelos de Cattell e Horn (teoria da inteligência fluida e cristalizada) e o mode- lo de Carroll (teoria dos três estratos), denominada Teoria Psicométrica das Habilidades Cognitivas de Cattell-Horn-Carroll (CHC).

No Brasil, os estudos sobre a inteligência são poucos, e os instrumentos usados para sua avaliação apresentam problemas psicométricos relacionados à validade (Sisto, Codenotti, Costa \& Nascimento, 1979; Noronha, Sartori, Freitas \& Ottati, 2001). Nesse contexto, o presente estudo tem como foco a busca de evidência de validade para o R-1: Teste não verbal de avaliação da inteligência Forma B, de autoria de Rynaldo de Oliveira, publicado pela Vetor Editora Psicopedagógica em 1973 (Oliveira, 1973). Esse teste foi proposto pelo autor para ser empregado no exame psicotécnico de motoristas, bem como em áreas que necessitem de um teste alternativo para pessoas analfabetas, com baixa escolaridade ou com dificuldades específicas para a compreensão do português.

De acordo com o manual do R-1 Forma B (Sisto, Santos \& Noronha, no prelo), foram realizados dois tipos de estudos para a verificação dos parâmetros psicométricos; em um deles, itens foram analisados com vistas a explicitar suas características subjacentes e, no outro, identificaramse os tipos de raciocínio implícito nos diferentes itens do teste. No primeiro estudo usou-se a análise fatorial e dois fatores foram constatados. Em um segundo estudo, objetivando conhecer as habilidades cognitivas envolvidas, os itens foram analisados qualitativamente com base nas leis que regem o fator $g$ (Spearman, 1927) e na Teoria de GfGc de Catell (1971).

A análise fatorial acusou a existência de dois fatores, sendo que o Fator 1 englobou, em maior quantidade, itens que podem depender de aprendizagem cultural e de peculiaridades do meio ambiente, ou que exigem como resposta igualdade, similaridade ou continuidade gestáltica; e, em menor quantidade, itens que requerem edução de relação e de correlatos. Em razão dessas características, o Fator 1 foi interpretado como assemelhado à inteligência cristalizada de Cattel (1971). Por sua vez, os itens do Fator 2 foram mais facilmente interpretáveis e mais específicos, pois tratam basicamente de edução de relações e de correlatos, o que estaria em consonância ao fatorg de Spearman.

Além desses estudos, o Manual fornece também duas pesquisas de evidências de validade, uma convergente e outra desenvolvimental. No 
Teste de inteligência R1 - forma B E G36: evidência de validade de critério

entanto, não fornece indicadores de evidências de validade de critério, em razão do que se propôs o presente estudo. Assim, o presente trabalho visa a analisar evidências de validade por grupos contrastantes, valendo-se da pontuação obtida no R1: Teste não verbal de avaliação da inteligência para a formação dos grupos.

\section{Método}

\section{Participantes}

Com base nessa coleta obteve-se informação de 78 alunos de Cursos de Educação de Jovens e Adultos, todos eles freqüentando o telecurso de segundo grau. Desses alunos, 27 (34,6\%) eram do sexo feminino e $51(65,4 \%)$ do gênero masculino. A idade mínima foi de 15 anos e a máxima de 64, com uma média de 36,84 e um desvio padrão de 10,10.

\section{Instrumentos}

O teste R1 - Forma B é constituído por 40 itens apresentados em um caderno, com folha de resposta à parte. Para cada questão há de 6 a 8 possibilidades de respostas. Sua correção implica em atribuir um ponto a cada resposta correta, sendo 40 o máximo de pontos possíveis.

O teste G-36, aqui utilizado, foi construído por Boccalandro (2003). É composto por 36 itens contidos num caderno, sendo a folha de respostas apresentada separadamente. Há 6 alternativas de respostas para cada questão e o escore máximo é de 36 pontos.

Os itens foram construídos com base no Teste de Matrizes Progressivas de Raven (Raven, Raven \& Court, 1991). Os primeiros estudos psicométricos apóiam-se em evidências de validade convergente, comparando seus resultados com os obtidos pela aplicação do Teste de Dominós (Anstey, 1963), tendo sido encontrada uma correlação de 0,84. A fidedignidade, analisada pelo método split-half, foi de 0,82. Outras evidências de validade foram apresentadas em 2003, quando o G36 foi aplicado simultaneamente com o Teste de Matrizes Progressivas de Raven e o índice de correlação obtido foi o de 0,76.

\section{Procedimento}

Após a devida autorização do Comitê de Ética, das escolas e dos participantes, deu-se início à coleta de dados. A aplicação do instrumento foi coletiva, em grupos de 15 a 20 sujeitos, sendo que em cada sala havia pelo menos um aplicador e um auxiliar. O tempo médio de aplicação foi de aproximadamente 50 minutos para cada instrumento.

\section{Resultados}

\section{a - grupos de contraste forma dos pela pontuação total do R1- Forma B}

Para constituir os dois grupos extremos com base na pontuação total do R1 - Forma B foram selecionados, dentre os 78 estudantes, $25 \%$ dos participantes com as maiores e outros $25 \%$ com as menores pontuações. Pelos dados da aplicação, o ponto de corte para o grupo extremo superior foi uma pontuação igual ou superior a 29 pontos; e para o grupo extremo inferior, igual ou inferior a 14 pontos.

No total, os grupos extremos ficaram constituídos por 41 pessoas. O grupo extremo superior foi constituído por 20 pessoas com idades entre 15-45 anos (média=34,29 e DP=7,74). Por sua vez, o grupo extremo inferior ficou com 21 pessoas com idades entre 18-64 anos (média $=41,10$ e $\mathrm{DP}=15,70)$. Com relação ao sexo, $35 \%$ eram do sexo feminino e $65 \%$ do masculino.

A diferença de médias entre os grupos extremos produzidos pelo R1- Forma B foi avaliada pelo $t$ de Student, com nível de significância de 0,05. Na Tabela 1 são apresentados os valores de t e p, além das pontuações mínima e máxima, média e desvio padrão do G-36 em relação aos grupos extremos.

As médias mostraram diferenças significativas pela prova aplicada. Os dados indicaram que a média do grupo extremo inferior foi bem menor $(6,60)$ que a média do grupo extremo superior $(17,48)$. Esse resultado permite concluir que os grupos extremos formados pelo R1 - Forma B são discriminados pelas pontuações do G-36. 


\section{Tabela 1. Valores de t e p, pontuações mínima e máx ima, média e desvio pa-} drão do G-36 em relação aos grupos ex tremos.

\begin{tabular}{ll|l|l|l|l|l}
\hline \multicolumn{1}{c|}{ Pontuação } & $\mathbf{t}$ & $\mathbf{p}$ & $\begin{array}{l}\text { Pontuação } \\
\text { Mínima }\end{array}$ & $\begin{array}{l}\text { Pontuação } \\
\text { Máxima }\end{array}$ & Média & $\begin{array}{l}\text { Desvio } \\
\text { Padrão }\end{array}$ \\
\hline $\begin{array}{l}\text { Grupo extremo inferior } \\
\text { Grupo extremo superior }\end{array}$ & $2,9,741$ & 0,000 & 2 & 15 & 6,60 & 3,66 \\
Escore total & 26 & 112,19 & 5,72 & 17,48 & 3,49 \\
\hline
\end{tabular}

\section{b- grupos de contraste formados pela pontuação dos Fatores 1 e 2 do R1- Forma B}

Foram realizadas análises levando em consideração os dois fatores do R1 - Forma B e, para tanto, foram formados grupos extremos para ambos os fatores. Com base no Fator 1, o grupo extremo inferior (25\% com menor pontuação, ou seja, até 10 pontos) ficou com 21 sujeitos e o grupo extremo superior (25\% com maior pontuação, ou seja, acima de 18 pontos) ficou com 20. Por sua vez, no Fator 2, o grupo extremo inferior (25\% com menor pontuação, ou seja, até 2 pontos) ficou com 21 sujeitos e o grupo extremo superior (25\% com maior pontuação, ou seja, acima de 11 pontos) ficou com 20.

As diferenças de médias entre os grupos extremos produzidos pelos fatores 1 e 2 do R1- Forma B foram avaliadas pelo tde Student, com nível de significância de 0,05. Na Tabela 2 são apresentados os valores de te $p$, além das pontuações mínima e máxima, média e desvio padrão do G-36 em relação aos grupos extremos, para ambos os fatores.

Novamente observou-se que os grupos extremos, para ambos os fatores, produziu médias que mostraram diferenças significativas pela prova aplicada. Para ambos os fatores, os dados indicaram que as médias dos grupos extremos inferiores foram bem menor (6,47 para o Fator 1 e 6,10 para o Fator 2) que as médias dos grupos extremos superiores $(15,77$ para o Fator 1 e 17,52 para o Fator 2). Esses resultados permitem concluir que os grupos extremos formados para os dois fatores do R1 - Forma B são discriminados pelas pontuações do G-36.

\section{Discussão e Considerações Fina is}

Dada a importância que a avaliação da inteligência tem na atuação do psicólogo e tendo em vista a necessidade de pesquisas que objetivem analisar as qualidades psicométricas dos instrumentos padronizados, o presente estudo procurou verificar evidências de validade do R-1: Testenão verbal de avaliação da inteligência, por meio de grupos contrastantes. Para isso, analisaram-se as diferenças de médias do G-36 Teste de Inteligência não Verbal em razão de grupos de pessoas com pontuações extremas, constituídas com base nas pontuações do R-1- Forma B.

\section{Tabela 2. Valores de te p, pontuações mínima e máx ima, média e desvio padrão do G-36 em relação a os grupos ex tremos para os fatores 1 e 2.}

\begin{tabular}{l|l|l|l|l|l|l}
\hline Medidas & $\mathbf{t}$ & $\mathbf{p}$ & \multicolumn{2}{|c|}{ Grupo ex tremo inferior } & \multicolumn{2}{c}{ Grupo extremo superior } \\
\cline { 4 - 7 } & & & Média & Desvio padrão & Média & Desvio padrão \\
\hline Fator 1 & $-8,23$ & 0,000 & 6,47 & 3,73 & 15,77 & 4,08 \\
Fator 2 & $-12,12$ & 0,000 & 6,10 & 2,57 & 17,52 & 3,39 \\
\hline
\end{tabular}


Teste de inteligência R1 - forma B E G36: evidência de validade de critério

Os resultados indicaram em duas análises diferentes, tanto na que se refere à pontuação geral dos participantes, quanto na que pontua o instrumento de acordo com seus fatores 1 e 2, que os grupos extremos são discriminados pela pontuação do G-36. De acordo com Anastasi e Urbina (2000), tal estudo permite trazer à luz reflexões acerca da validade de critério por grupos contrastantes, uma vez que o teste R-1-Forma B usado como critério para formar grupos de altas e baixas pontuações discriminou também as médias altas e baixas do teste G36. De certa forma, com base no fato de que um teste serviu de critério para o outro, é possível interpretar que há evidências mútuas de validade para discriminar a tendência a obter pontuações altas e baixas em ambos os testes. Em outros termos, há uma possibilidade de erro pequena, no sentido de uma pessoa obter classificações altas ou baixas em um teste e não obter em outro.

Nesse sentido, vale lembrar que o R1 Teste não Verbal Forma B foi interpretado como possuindo um fator assemelhado à inteligência cristalizada (Cattell, 1971) e, outro, ao fator g de Spearman. Ao lado disso, o G-36 foi fundamentado teoricamente no fator g. Sua construção teve como base o Teste de Matrizes Progressivas de Raven (Raven, Raven \& Court, 1991), conforme informa seu manual. Em razão dos dados encontrados neste estudo, é possível questionar se o G-36 é unidimensional e se apenas o fator g é necessário para responder o instrumento como um todo, como está proposto em seu Manual.

Embora os resultados desse estudo tenham permitido compreender que os grupos extremos foram discriminados pela outra medida utilizada e, dessa forma, tenha fornecido evidência de validade, recomenda-se a importância de se realizar outros estudos com amostras diferentes, comparando os instrumentos com outros critérios ou utilizando-se da comparação com outro teste. Tais estudos contribuirão com o avanço das pesquisas na área de avaliação psicológica, assim como com a qualidade dos seus instrumentos.

\section{Referências}

Almeida, L.S. (1988). Teorias da inteligência. Porto: Edições Jornal de Psicologia.

Anastasi, A., \& Urbina, S. (2000). Testagem Psicológica. Porto Alegre: Artes Médicas.

Anstey, E. (1963). Test de Dominós. Manual. Buenos Aires: Paidós.

Carroll, J.B. (1993). Human cognitive abilities. New York: Cambridge University Press.

Cattell, R. B. (1971). Abilities: Their structure, growth, and action. Boston: Houghton Mifflin.

Cattell, R. B. (1992). The relevance of fluid and crystallized intelligence concepts to nature-nurture investigation. The Manking Quartely, 23, 359375.

Flanagan, D. P., McGrew, K. S., \& Ortiz, S. O. (2000). The Weschsler Intelligence Scales and CHC Theory: A contemporany approach to interpretation. Boston: Allyn \& Bacon.

Horn, J. L. (1991). Measurement of Intellectual Capabilities: a review of theory. In K. S. McGrew, J. K. Werder, \& R. W. Woodcock. Woodcock-Johnson technical manual (pp. 197-232). Chicago: Riverside.

Noronha, A. P. P.; Sartori, F. A.; Freitas, F. A., \& Ottati, F. (2001). Informações contidas nos manuais dos testes: Um estudo com testes de inteligência. Psicologia em Estudo, 6, 195-202.

Raven, J., Raven, J.C., \& Court, J.H. (1991). Manual for Raven's Matrices e Vocabulary Scales: Section 1. General overview. Oxford: UK: Oxford Psychologists Press.

Schelini, P.W. (2000). Bateria multidimensional de inteligência infantil: Proposta de instrumento. Tese de Doutorado. PUC-Campinas. Campinas-SP.

Sisto, F. F., Santos, A. A. A., \& Noronha, A. P. P. (no prelo). R-1: Teste Não Verbal de Inteligência Forma B - Manual. São Paulo: Vetor.

Sisto, F. F., Codenotti, N., Costa, C. A. J., \& Nascimento, T. C. N. (1979). Testes Psicológicos no Brasil: que medem realmente? Educação e Sociedade, $1,152-165$. 
Spearman, C. (1927). Las Habilidades del Hombre: su naturaleza y medición. Buenos Aires: Paidós.

Sternberg, R. J. \& Powell, J. S. (1983). The development of intelligence. Em P.H. Mussen (Org.), Handbook of child psychology (pp. 341-419). New York: John Wiley \& Sons.
Thurstone, L. L. (1938). Primary mental abilities. Chicago: University of Chicago Press.

Recebido em/Received in: 16/11/2004

Aprovado em/Approved in: 15/03/2005 